\title{
Bone marrow granulomatosis: two case reports
}

\author{
Giacomo Andreani, ${ }^{1}$ Alessandro Morotti, ${ }^{1}$ Matteo Dragani, ${ }^{1}$ Francesca Caciolli, ${ }^{1}$ Bruna Crea, ${ }^{1}$ Valerio Marci, ${ }^{2}$ \\ Ubaldo Familiari, ${ }^{2}$ Angelo Guerrasio ${ }^{1}$
}

${ }^{1}$ Department of Clinical and Biological Sciences, University of Turin, San Luigi Gonzaga Hospital; ${ }^{2}$ Department of Pathology, San Luigi Gonzaga Hospital, Orbassano (TO), Italy

\begin{abstract}
Bone marrow granulomatosis is not a common finding and remains a diagnostic challenge. Here below we report two bizarre but not so rare cases of bone marrow granulomatosis: bone marrow involvement by Mycobacterium avium-intracellulare and reaction to intravesical administration of Bacillus Calmette-Guérin for non-muscle-invasive bladder cancer; two distinct culprits suggesting the wide range of possible causative factors of this histopathological pattern. In the discussion we provide a brief overview of causes and suggestions for a diagnostic approach.
\end{abstract}

\section{Case Reports}

\section{Case \#1}

A 71-year-old man was referred to our Department for pancytopenia with the suspect of a hematological malignancy. Physical examination was negative, lymph nodes, liver and spleen were not enlarged. Complete blood count (CBC) upon admission: white blood cells count (WBC) 1400/uL, hemoglobin (Hb) $9.0 \mathrm{~g} / \mathrm{dL}$, platelets 2000/uL; furthermore fibrinogen resulted consumed $(84 \mathrm{mg} / \mathrm{dL}$; normal value $>150 \mathrm{mg} / \mathrm{dL}$ ) with normal prothrombin time and activated partial thromboplastin time, cholestatic indices were elevated

Correspondence: Giacomo Andreani, Department of Clinical and Biological Sciences, University of Turin, San Luigi Gonzaga Hospital, Regione Gonzole 10, 10043 Orbassano (TO), Italy.

Tel.: +39.011.9026305 - Fax: +39.011 .9026963 .

E-mail: giacomo.andreani@unito.it

Key words: Bacillus Calmette-Guérin; bone marrow granulomatosis; mycobacteria; metallosis; pancytopenia.

Contributions: GA, AM, contributed equally to this work.

Conflict of interests: the authors declare no potential conflict of interests.

Received for publication: 12 February 2019.

Accepted for publication: 3 June 2019.

This work is licensed under a Creative Commons Attribution NonCommercial 4.0 License (CC BY-NC 4.0).

${ }^{\circ}$ Copyright: the Author(s), 2019

Licensee PAGEPress, Italy

Italian Journal of Medicine 2019; 13:181-184

doi:10.4081/itjm.2019.1150 (gamma-glutamyl transferase $239 \mathrm{U} / \mathrm{L}$; alkaline phosphatase $305 \mathrm{U} / \mathrm{L}$ ). No fever history was reported. He was well-oriented in time and space but anamnestic collection was difficult because of a state of agitation. A remote pathological anamnesis reported story of hypertension and dyslipidemia, a bilateral hip arthroplasty eight years before, coronary-artery bypass graft surgery for acute myocardial infarction six years before, transurethral resection of prostate for benign prostatic hyperplasia and a major depressive episode after the death of his brother few months before admission. Serological tests for hepatitis A, B, and C viruses, EpsteinBarr virus (EBV), cytomegalovirus (CMV) and human immunodeficiency virus (HIV) were negative.

Both bone marrow biopsy and aspiration were performed, but results were negative for hematologic neoplasia. No blast cells were observed but clusters of histiocytes with microgranulomatosis and multinucleated giant cells were documented by pathologist (Figure 1A). Positron emission tomography and neck-thorax-abdomen computed tomography (CT) with contrast dye gave negative results. Echocardiographic assessment was regular.

Considering the absence of leukemic cells and the presence of granulomas on bone marrow, we focused our diagnostic investigation on the research of an infectious agent. Widal-Wright test (a test for the serodiagnosis of infections caused by Salmonella typhi, Salmonella enterica, Brucella melitensis), serology for leishmaniasis, serum polymerase chain reaction (PCR) for EBV, CMV, human herpesvirus-8, were all negatives; PCR for Tropheryma whipplei in feces was negative. A new bone marrow biopsy (20 days after the first one) confirmed the picture of giant-cell granulomatosis. On that occasion a real time PCR for $M y$ cobacterium tuberculosis was carried out on bone marrow sample but the result was negative; a specimen was sent for culture. 
Tests for anti-nuclear, anti-dsDNA, anti-neutrophil cytoplasmic, anti-extractable nuclear antibodies and rheumatoid factor were all negatives.

Day after day the patient became more nervous and sleepless; talking with him was almost impossible because of his state of agitation. A major depressive episode was diagnosed by the psychiatric consultant. A metallosis caused by circulating particles originated from ceramic-on-metal hip arthroplasty consumption was suspected (considering psychiatric presentation and tremors at rest too): thus cobalt, chromium, and molybdenum were dosed in blood but all resulted within normal ranges; moreover, hair mineral analysis was negative for metal intoxication.

The patient was discharged after 40 days of hospitalization without a precise diagnosis. Since infectious noxae were considered improbable, steroid therapy was started (prednisone $1 \mathrm{mg} / \mathrm{kg} / \mathrm{die}$ ). At outpatient follow-up he felt better physically and psychologically. The $\mathrm{CBC}$ improved and fibrinogen rose to normal values: WBC 1600/uL (neutrophils 1220/uL); Hb $10.1 \mathrm{~g} / \mathrm{dL}$; platelets 50,000; fibrinogen $133 \mathrm{mg} / \mathrm{dL}$ (14 days after discharge). Steroid therapy was continued.

Two months after discharge we received the result of the bone marrow culture: a positivity for M. aviumintracellulare was reported; but patient had already died few days before for intracranial hemorrhage following an accidental fall from stairs at home.

\section{Case \#2}

An 82-year-old man was admitted to our department for fever and pancytopenia. He was affected by dilated cardiomyopathy for over 10 years and implantable cardioverter-defibrillator was placed seven years before; residual left ejection fraction was 35\%. Few months before this hospitalization he underwent a surgical intervention for a femoral fracture. On that occasion non-muscle-invasive bladder cancer was diagnosed; therapy with intravesical Bacillus CalmetteGuérin (BCG) instillation was started (6 consecutive weekly doses) after transurethral bladder resection.

The CBC at the admission: WBC 2390/uL (neutrophils 1680/uL); Hb $10 \mathrm{~g} / \mathrm{dL}$; platelets 76,000/uL. Chest $\mathrm{x}$-ray was negative. Wide spectrum antibiotic and low dose steroid therapy were started.

Bone marrow biopsy revealed the absence of blasts but the presence of microgranulomas (Figure 1B). Screening for infectious agents (as for Patient 1) was negative. Myeloculture was negative for $M$. $t u-$ berculosis and for atypical mycobacteria. Patient was discharged afebrile, with an improved CBC compared to admission. A diagnosis of suspected BCGitis with bone marrow involvement was made. No specific therapy was administered. Follow-up was regular with clinical improvement.

\section{Discussion}

Bone marrow granulomatosis is a challenging finding. Patients with this histological pattern are generally admitted for pancytopenia and fever. Obviously, a hematological clonal disorder should be excluded first by adequate bone marrow examination. Concerning the differential diagnosis of granulomatosis, infectious agents are probably the first causative agents to be investigated. Mycobacteria infections are the most notorious culprits and the first step for diagnosis is to distinguish caseating (wherein M. tuberculosis is gen-

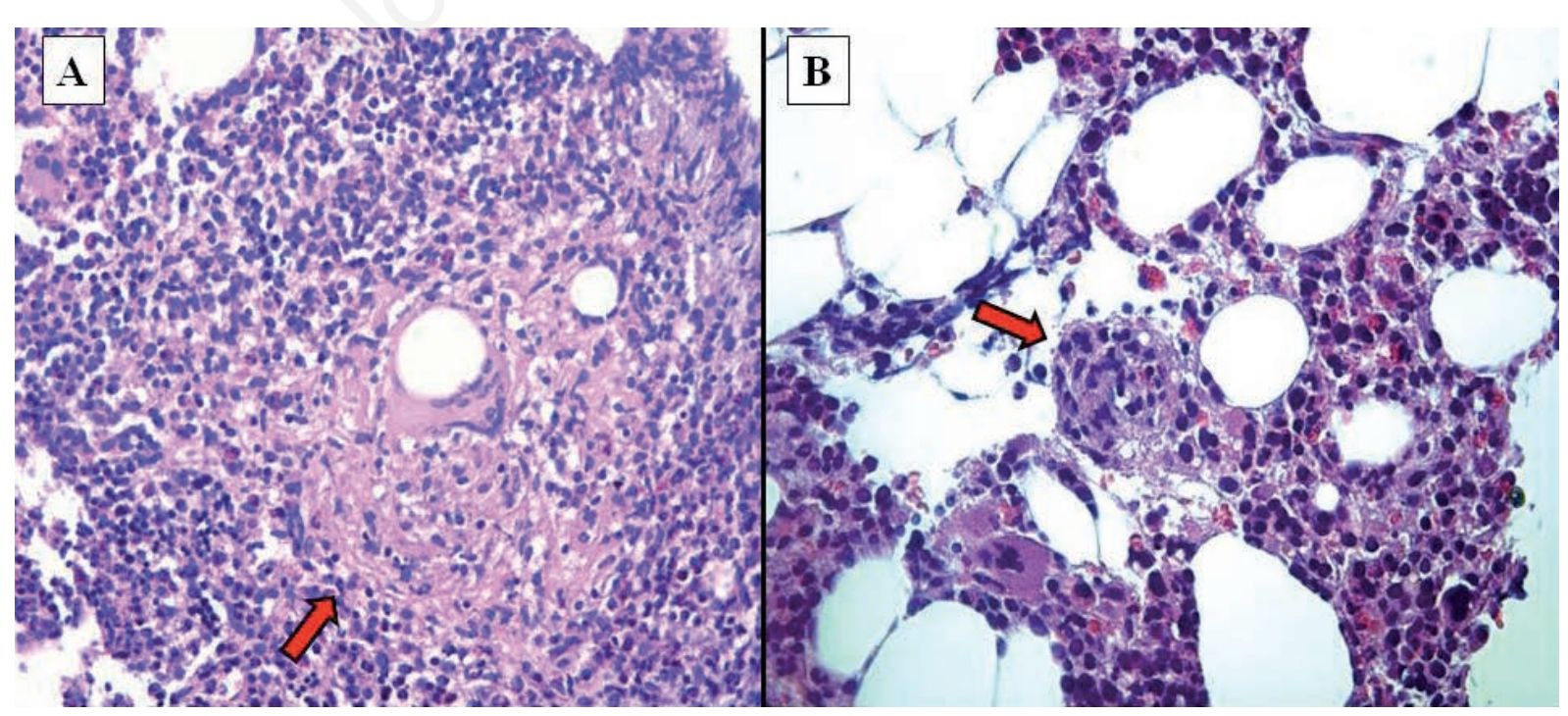

Figure 1. Bone marrow granulomatosis: case report \#1 (A) and \#2 (B). 
erally the culprit) from non-caseating granulomas. There are more than 170 identified species of mycobacteria but only a minority of species are obligate human pathogens, the remaining ones generally cause illness when host defenses are reduced. ${ }^{1}$ Obligate human pathogens include $M$. leprae (causative of leprosy) and organisms of the M. tuberculosis complex: M. tuberculosis, M. africanum, M. bovis and BCG. M. kansasii and M. avium-intracellulare can cause opportunistic infections usually in immunosuppressed patients (e.g. HIVinfected patients, transplant patients, patients with inherited cellular immunodeficiencies). ${ }^{2}$ Each one of these mycobacteria can be the primum movens for inducing granulomas in bone marrow and in other more usual sites (lung, lymph nodes, spleen, liver, etc.).

A curious, iatrogenic and maybe underestimated cause of granulomatosis is the so-called BCGitis or BCGosis. BCG is an attenuated live strain of M. bovis that is used as a vaccine in countries were tuberculosis is endemic. Moreover, sequential intravesical administration of BCG is also the gold standard treatment for intermediate and high-risk non-muscle-invasive bladder cancer after transurethral bladder resection. ${ }^{3}$ Many case reports have been published on the extensive granulomatosis of kidneys, liver, spleen, lungs and bone marrow as possible consequences of BCG bladder instillations. ${ }^{4,5}$ Toxicity is reported to be dose-dependent and strain-specific. ${ }^{6}$ Antituberculous drugs administration and low-dose steroid treatment are suggested as empirical therapy in suspected cases. ${ }^{4}$ Despite scanty literature data, other infectious causes of bone marrow granulomatosis in patients presenting with pancytopenia and fever include Leishmania, Brucella, Coxiella burnetii (Q fever), Bartonella, EBV, CMV.?

Another bizarre but more than anecdotal cause of tissue (and bone marrow) granulomatosis is a physiological reaction to foreign-bodies released from arthroplastic materials. ${ }^{8-12}$ Different types of hip and knee implants have been used successfully in orthopedics for years despite they are known to release metallic wear particles causing local muscle and bone reaction that can then leach into bloodstream spreading to lymph nodes, spleen, liver, heart, lungs, kidneys, thyroid, central nervous system with distinct pictures of presentation, defining the diagnosis of metallosis. Hip radiography, CT and/or magnetic resonance are advised to document possible prosthesis defects. It is important to know the type of materials of prosthetic implants (polyethylene, titanium, cobalt-chromium-molybdenum alloys); dosing these materials in blood, urine, sputum and a hair mineral analysis are recommended in suspected cases. Metal intoxication generally happens years after device implantation as a consequence of friction between two surfaces of the joint. Hip replacement intervention with drainage of the involved site is mandatory; utilization of antidotes against culprit circulating materials are suggested if available (e.g. 2,3dimercaptopropane-1-sulfonate for cobalt intoxication).

Potentially all agents that cause granulomatosis can induce a bone marrow involvement, from berylliosis (an occupational lung disease) to sarcoidosis ${ }^{13}$ and all these causes have to be taken into consideration. Granulomatous bone marrow reactions have also been described in hematological malignancies, especially in Hodgkin and non-Hodgkin lymphomas but it is not clear if these findings are related to malignancy or infections (Table 1). ${ }^{14-16}$

\section{Conclusions}

Granulomatosis is a characteristic pattern of chronic inflammation observed as response to a persistent antigenic stimulus characterized by aggregates of macrophages, multinucleated giant cells, histiocytes with or without caseous necrosis. Bone marrow granulomatosis is not a common report. Careful anamnestic collection, accurate physical examination and routine blood test can help us to continue diagnostic investigation; certainly, infectious causes have to be ruled out, above all mycobacteria infections, in particular we recommend myeloculture after bone marrow aspiration.

Table 1. Non-exhaustive summary of possible causes of bone marrow granulomatosis.

Infectious causes

Mycobacteria (M. tuberculosis, M. bovis, Bacillus Calmette-Guérin, M. leprae, M. avium-intracellulare, M. kansasii, etc.), Leishmania, Brucella, Coxiella, Bartonella, Epstein-Barr virus, cytomegalovirus, fungal infections

Foreign-bodies reaction

Metallosis (occupational and environmental exposure, e.g. berylliosis), arthroplastic materials (cobalt, chromium, etc.)

Autoimmune causes

Vasculitis, sarcoidosis

Hematological malignancies

Hodgkin lymphoma, non-Hodgkin lymphoma

Drugs $^{16}$

Amiodarone, ibuprofen, phenytoin, allopurinol, sulfasalazine 


\section{References}

1. Fedrizzi T, Meehan CJ, Grottola A, et al. Genomic characterization of nontuberculous mycobacteria. Sci Rep 2017;7:45258.

2. Guglielmetti L, Mougari F, Lopes A, et al. Human infections due to nontuberculous mycobacteria: the infectious diseases and clinical microbiology specialists' point of view. Future Microbiol 2015;10:1467-83.

3. Tran-Harding K, Nair RT, Ganesh H. Renal granulomatosis post intravesical bacillus Calmette-Guerin therapy for non-muscle-invasive bladder cancer. J Clin Imaging Sci 2018;8:18.

4. Schattner A, Gilad A, Cohen J. Systemic granulomatosis and hypercalcaemia following intravesical bacillus Calmett-Guérin immunotherapy. J Intern Med 2002;251: 272-7.

5. Lamm DL. Efficacy and safety of bacilli CalmetteGuerin immunotherapy in superficial bladder cancer. Clin Infect Dis 2000;31:S86-90.

6. Mohammed A, Arastu Z. Emerging concepts and spectrum of renal injury following intravesical BCG for nonmuscle invasive bladder cancer. BMC Urol 2017;17:114.

7. Keynan Y, Yakirevitch E, Shusterman T, et al. Bone marrow and skin granulomatosis in a patient with Bartonella infection. J Med Microbiol 2007;56:133-5.

8. Péoc'h M, Moulin C, Pasquier B. Systemic granulomatous reaction to a foreign body after hip replacement. $\mathrm{N}$
Eng J Med 1996;335:133-4.

9. Urban RM, Jacobs JJ, Tomlinson MJ, et al. Dissemination of wear particles to the liver, spleen, and abdomen lymph nodes of patients with hip or knee replacement. J Bone Joint Surg Am 2000;82:457-76.

10. Cohen D. How safe are metal-on-metal hip implants? BMJ 2012;344:e1410.

11. Allen LA, Ambardekar AV, Devaraj KM. Missing elements of the history. N Eng J Med 2014;370:559-66.

12. Balbouzis T, Georgiadis T, Grigoris P. Granulomatous lung disease: a novel complication following metallosis from hip arthroplasty. Hip Pelvis 2016;28:249-53.

13. Zhou Y, Lower EE, Li H, et al. Clinical characteristics of patients with bone sarcoidosis. Semin Arthritis Rheum 2017;47:143-8.

14. Brunner A, Kantner J, Tzankov A. Granulomatous reactions cause symptoms or clinical imitate treatment resistance in small lymphocytic lymphoma/chronic lymphocytic leukaemia more frequently than in other non-Hodgkin lymphomas. J Clin Pathol 2005;58:815-9.

15. Kumar-Kandala Jeevan S, Paul-Tara R, Uppin S, Uppin $\mathrm{M}$. Bone marrow granulomas: a retrospective study of 47 cases (a single center experience). Am J Int Med 2014;2:90-4.

16. Mukhopadhyay S, Mukhopadhyay S, Abraham NZ Jr, et al. Unexplained bone marrow granulomas: is amiodarone the culprit? A report of 2 cases. Am J Hematol 2004;75:110-2. 